\title{
Der klinische Alltag in einer COVID-19-Intensivstation
}

Lisa Pongratz, befragt von Wolfgang Kröll

Kröll: Liebe Lisa! Danke für deine Bereitschaft mir für den zweiten Band des Buches „Die Corona-Pandemie“ einige Fragen zu deiner Tätigkeit und deiner Sicht der Dinge im Gesundheitswesen zu beantworten:

$D u$ arbeitest als diplomierte Gesundheits- und Krankenpflegerin (DGKP) auf einer chirurgischen Intensivstation, die sich auf die Behandlung und Betreuung von Patienten spezialisiert hat, die mit SARS-CoV2 infiziert sind? Wie geht es dir und deinen Kolleginnen dabei?

Pongratz:Als die vielen beklemmenden Horrorszenarien aus China medial kolportiert wurden, und das ungeheure Ausmaß der rasanten Virusverbreitung sich zu einer Pandemie entwickelte, war mir klar, dass auch Österreich davon betroffen sein wird. Unsere Station ist spezialisiert auf die intensivmedizinische Behandlung von chirurgischen Patientinnen unterschiedlichster Disziplinen, mit hohen Belegzahlen, ausgestattet mit 10 Beatmungspositionen, davon 2 Isolierkojen. Aufgrund der baulich isolierten Lage in einem Innenhof, erwies sich unsere Station zur Behandlung von COVID-19-positiven PatientInnen mit Intensivpflegebedarf als strategisch günstig. Nach anfänglich gemischten Übergangslösungen und im engen Austausch mit den anderen Intensivstationen wurde unsere Station Anfang November als SARS-CoV2 positive Intensivisoliereinheit ausgewiesen. Die unmittelbare Konfrontation mit einem neuartigen Virus, das Gefühl unmittelbar „an der Front zu sein “, machte das Team betroffen und erzeugte auch bei uns große Unsicherheit und Anspannung: einerseits war da Angst vor Ansteckung, um die eigene Gesundheit, der Familie, des privaten Umfelds, werden wir die Anforderungen bewältigen? Auf der anderen Seite konnten wir im Gegensatz zu anderen Stationen von vornherein das Risiko einschätzen, wir nahmen ausschließlich SARS-CoV2-positiv getestete Patientinnen auf. 
Kröll: Gibt es bzw. hat es bei euch Mitarbeiter gegeben, die sich infiziert haben und die an COVID-19 erkrankt sind? Sind diese schon wieder genesen oder leiden sie noch an den Folgen dieser Erkrankung? Wie geht es Dir persönlich bzw. wie geht es deinem privaten und familiären Umfeld? Gibt es in diesem Bereich infizierte Familienmitglieder? Bist du geimpft? Und wie hast du die Impfung vertragen, hattest du irgendwelche Impfreaktionen? Du hast dich bis jetzt nicht infiziert; achtest du mehr auf deinen eigenen Schutz, mehr auf deine eigene Hygiene als jene Mitarbeiterinnen, die an COVID-19 erkrankt sind? Die fehlende Bereitschaft Hygienemaßnahmen einzuhalten, ist das aus deiner Sicht einfach ein schlampiges Verhalten oder ist es eine Nicht-Akzeptanz dieser Maßnabmen als sinnvoll?

Pongratz: Wir waren und sind als SARS-CoV2-positive Intensivstation privilegiert, was den Impfzeitpunkt und die Personaltestungen betreffen. Ich durfte die betriebliche Gelegenheit zur Impfung schon im Jänner 2021 nützen, nur begleitet von lokal auf den Oberarm beschränkten geringen Schmerzen bis zum 2. Tag nach der Impfung. Ich war einfach nur erleichtert und fühle mich durch die Impfung, den regelmäßigen PCR Testungen und der Schutzkleidung beruflich optimal geschützt.

Auf der eigenen Station gibt es nur einen geringen Anteil an infizierten MitarbeiterInnen. Gemäß der sehr strengen Hygienerichtlinien und dem Bemühen um deren Einhaltung aller im Team, kann davon ausgegangen werden, sich dienstlich nicht angesteckt zu haben. Zum Teil nur leicht erkrankt oder asymptomatisch, sind alle genesen, meines Wissens ohne Einschränkungen.

Die Möglichkeit einer Ansteckung besteht meiner Meinung nach eher im privaten Umfeld, in der Öffentlichkeit, wo man sich über den ignoranten und teils sorg-, und verantwortungslosen Umgang mancher Mitmenschen nur wundern kann! So sind Ansteckungen und Clusterbildungen möglich. Da gilt es, vorsichtig zu bleiben.

Ich habe auch die organisatorische Problematik im Bereich der 24-Stundenpflege im Rahmen der Betreuung meiner 92-jährigen Mutter kennengelernt. Da lernt man den hohen Standard des eigenen Gesundheitssystems erst richtig schätzen! Die Empfehlungen der Experten zu befolgen, sind für mich selbstverständlich! Dazu zählen zum Beispiel auch regelmäBige Antigentests und sich impfen zu lassen aus Riesenrespekt vor der Gesundheit meiner Mitmenschen. Ähnlich verhält sich auch mein enges privates Umfeld, das bisher Gott sei Dank vor Ansteckungen verschont geblieben ist. 
Kröll: Die Corona-Pandemie ist über Europa relativ plötzlich und nicht ganz vorhersehbar hereingebrochen. Wie war die Intensivstation darauf vorbereitet? Wurden euch ausreichend Schutzanzüge, Schutzbrillen und Masken von Anfang an zur Verfügung gestellt oder gab es dabei irgendwelche Probleme und Mängel? Haben notwendige Utensilien (Masken, Schutzanzüge, Desinfektionsmittel etc.) gefehlt bzw. waren sie nur in eingeschränkter Form verfügbar?

Pongratz: Auch wenn sich die Betreuung und Behandlung von IntensivpatientInnen wesentlich von der Betreuung und Behandlung von PatientInnen auf einer Normalstation unterscheidet, haben wir alle eine solche Situation, nämlich mit Schutzausrüstungen zu arbeiten, noch nicht erlebt - zumindest nicht auf chirurgisch-anästhesiologischen Intensivstationen. Die Vorbereitung auf die Pandemie ging rasch und in Schritten vor sich: Routineeingriffe wurden abgesagt, Räumlichkeiten, ganze Stationen, Gebäude wurden adaptiert; das Personal wurde informiert via E-Mail und autorisierten Whats-App-Gruppen; regelmäßig fanden Schulungen statt, Krisenstäbe mit täglichen Upgrades waren an der Tagesordnung und Videokonferenzen zur raschen und kontaktlosen Informationsweitergabe wurden angeboten. Unsere Stationsleitung war rund um die Uhr erreichbar, unermüdlich am Organisieren, um sich und uns über aktuelle Arbeitsanweisungen zu informieren. Auch wir als Team versuchten unser Bestes $\mathrm{zu}$ geben und alle Anordnungen umzusetzen.

Am 21. März 2020 dann der erste Lockdown. Alles stand zum ersten Mal still: die Stadt, das Land, die Wirtschaft, die Gesellschaft. Social Distancing war angeordnet und die Krankenanstalten befanden sich in Warteposition.

Menschenleere Gänge in einem sonst pulsierenden Zentralkrankenhaus. Security an den Eingängen, Zugangsbeschränkungen überall. Gruselig. Anonyme, maskierte Gesichter. Keine sichtbare Mimik, die Kommunikation und die Wahrnehmung sind erschwert durch das ständige Tragen von Schutzmasken.

Strenge Hygienemaßnahmen mit jedem Schritt und Tritt, bei jeder Tätigkeit. Die Beschaffung ausreichender Schutzausrüstung war für den Betrieb sicherlich eine logistische Höchstleistung unter der anfänglich allgemein herrschenden Knappheit an Versorgungsmaterial. Unser persönlicher Schutz als „Systemerhalter“ war jedoch immer ausreichend sichergestellt.

Die Arbeit in Schutzkleidung ist im Umfeld einer Intensivbehandlung ja nichts Außergewöhnliches, die Einhaltung von Hygienerichtlinien am Intensivbett ist selbstverständlich und eine Bedingung am Krankenbett 
und dessen Umfeld. Die besonderen Umstände mit stundenlangem Eingehüllt-Sein in Kunststoff von Kopf bis Fuß, enganliegenden FFP 2/3 Mundmasken und Schutzbrillen als ständige Begleiter über die gesamte Dienstzeit von bis zu 12,5 Stunden, zehrt aber an der körperlichen Belastbarkeit: der Mund ist ständig trocken. Nach kurzer Zeit laufen manche Brillen an, schwitzen und Durst wird zur logistischen Herausforderung. Der Wechsel der Schutzausrüstung ist bei jedem/jeder PatientIn notwendig, beim Gang auf die Toilette. Nach einer kontinuierlichen Dienstfolge von mehreren Tagen ist das körperlich spürbar und benötigt längere Regenerationszeiten als üblich.

Es gibt strenge Pausenregelungen, nur 4 Personen dürfen gleichzeitig im Sozialraum während der Nahrungsaufnahme anwesend sein. Das braucht zeitliche und räumliche Adaptierung. Nebenbei entstehen Unmengen an Müll. Die Bereitstellung von waschbaren OP- Mänteln brachte erfreulicherweise Erleichterung durch einen besseren Tragekomfort und geringeren Müllmengen.

Mittlerweile sind wir routinierter im Umgang mit den Utensilien, und auch in der Betreuung von an COVID-19 erkrankten Personen. Die sinkende Infektionsrate lässt auf erste Impferfolge und einen Rückgang der Pandemie hoffen. Das Virus selbst wird sich einbürgern, aber hoffentlich berechenbar werden.

Kröll: Wie hast du generell die Zeit der Pandemie an deinem Arbeitsplatz erlebt? Waren die Arbeitsbedingungen für dich und deine Kolleginnen akzeptabel? Wie ist es Dir und deinen Kolleginnen bei der doch sehr aufwendigen Betreuung von Intensivpatienten ergangen?

Pongratz: Der Umstieg auf eine rein positive SARS-CoV2-Station brachte Veränderungen mit sich: lange Behandlungsdauer aufgrund der langen Isolierpflicht und der Schwere der Krankheitsverläufe. Ein Umstand, der alle im Team immer wieder sehr betroffen macht. Im Gegensatz zur ersten Welle, wo ein Großteil der Erkrankten um das 80.igste Lebensjahr lag, betrifft es in der zweiten Welle jüngere Patienten, 60 plus und jünger, zum Teil auch ohne Vorerkrankungen. Vor allem aber der Verlauf macht betroffen:

Die Viruserkrankung fordert von den Kranken enorm viel Kraft. Sie leiden an ATEMNOT bei jeder ihrer Aktivitäten, beim Aufsitzen, Essen, Trinken, Sprechen und allen notwendigen Pflegeinterventionen. Damit verbunden Angst, auch Panik. Die schlechte Oxygenierung nehmen die erkrankten Personen subjektiv nicht immer wahr, deswegen lassen sie sich erst oft (zu) spät behandeln. 
Die schnell eingreifende Therapie versucht durch gezielte und rasche invasive Therapiemaßnahmen die noch vorhandenen Ressourcen der PatientInnen zu bewahren und stärken, um ein Fortschreiten der Pneumonie aufzuhalten. Alle PatientInnen tun ihr Bestes! Manche schaffen es und genesen, und sind trotz aller Anstrengung äußerst motiviert. Bewundernswert!

Viele Erkrankte aber atmen um ihr Leben, bis ihnen trotz allem buchstäblich die Luft ausgeht. Der Körper hat die Energiereserven aufgebraucht und kann nicht mehr.

Nicht-invasive Beatmungstherapien, Mobilisation was möglich ist, Intubation, Tracheotomie, Bauchlagerungen sind an der Tagesordnung, invasive Therapien und Zugänge werden notwendig. Nach anfänglich scheinbar stabilen Verlaufsphasen und Besserungen schreitet die Erkrankung voran und endet nach langer und aufwändigster Behandlung trotz allem tödlich oder in einer schwer beeinträchtigenden Form. Für das gesamte betreuende Team immer eine große Frustration, da sich solche drastischen Verläufe häufig wiederholen.

Umso schöner, wenn PatientInnen positive Fortschritte machen und genesen.

Kröll: Würdest du bzw. deine KollegInnen auch psychologisch unterstützt, falls es erforderlich war? Von wem ist die Initiative dazu ausgegangen? Und wer hat euch betreut?

Pongratz: Ja, die Möglichkeit und Initiative ging vom Dienstgeber aus und kann in einem anonymen, geschützten Umfeld durch eine betriebsinterne Psychologin genützt werden. Zudem ist unsere Stationsleitung ständig bemüht, unser Team in allen Belangen zu unterstützen und Vorkommnisse in persönlichen Gesprächen und im Rahmen der täglichen Dienstübergaben zu reflektieren.

Wir als Intensivpersonal werden in vielen theoretischen Stunden für den Extrembereich ausgebildet, und jede Person, die sich für den Beruf entscheidet, sieht das als positive Herausforderung. Mit Notsituationen umzugehen, wird erst im ganz normalen „Intensivalltagswahnsinn“ erlernt: wahrnehmen, relevante Beobachtungen weitergeben, delegieren, entscheiden, unterstützen, informieren. Versiert und gewissenhaft sein in der Handhabung hochwirksamer Medikamente, von Medizinprodukte und Apparaturen.

Teamgeist, Durchführungsverantwortung, Eingreifen mit fachlicher Kompetenz, Herz und Empathie in den unterschiedlichen Phasen eines 
Intensivaufenthaltes; vermitteln zwischen Patientin/ Arzt / Angehörigen. Brücke zur Außenwelt sein; Trost spenden, begleiten im Sterbeprozess.

Beruhigen, Sicherheit vermitteln in einer apparatedominierten und befremdlichen Umgebung. Das schafft Vertrauen und dient als Basis eines Beziehungsaufbaus Pflegeperson/PatientIn, auch als Anlass für die Schwererkrankten trotz schwieriger Umstände mitzuarbeiten.

Verständnis dem kranken Menschen entgegenbringen, ihn/sie als Persönlichkeit mit all ihren Facetten wahrnehmen; Bedürfnisse erkennen und darauf eingehen. Sie da abholen, wo sie gerade sind. Und immer wieder motivieren; auch wenn die Person unruhig, in der Wahrnehmung eingeschränkt oder desorientiert ist, manches oder alles ablehnt und unkooperativ bleibt, oft über Tage hinweg.

Die Person hinter der Diagnose nicht zu vergessen in ihrer Gesamtheit! Das gibt mir Sinn und berührt im Umgang mit schwerkranken Menschen. All das bedeutet Intensivpflege.

Die Berufs- und Lebenserfahrung hilft, Prioritäten zu erkennen. Die technischen und therapeutischen Anforderungen und deren Dokumentation werden immer komplexer und zeitintensiver. Die Kunst bleibt es, mit den eigenen Ressourcen achtsam umzugehen, einen passenden Ausgleich zu finden, ohne auszubrennen. Das ist und bleibt auch immer ein persönlicher Lernprozess. Es gibt unterschiedliche persönliche Zugänge: Sport, ausspannen. Im Dienst reflektieren, evaluieren durch viele Gespräche, Arbeitskreise, Erfahrungsaustausch, „Neue“ einschulen. Offen sein für Neues. Krisen bergen auch immer Chancen für neue Wege.

In der Gruppe achten, dass der einzelne nicht überfordert wird. Auch Humor darf erlaubt sein. Unser schon immer starker Teamgeist ist ungebrochen und sicher einer der Gründe, dass auf unserer Station eine Personalfluktuation so gut wie nicht vorhanden ist.

Wenn Schwierigkeiten überwunden werden können in einem wertgeschätzten Umfeld, ist auch hoher Stresslevel, kurzfristige Personalknappheit besser zu bewältigen. Dann ist Pflege ein sehr befriedigendes Arbeitsfeld

Positive, wertschätzende Rückmeldungen im Team und der Vorgesetzten sind das Eine; tiefe, herzliche Dankbarkeit von Patientinnen und Angehörigen erleben das Andere.

Es lässt einen weitermachen und bedeutet letztendlich auch ein bisschen Glück. 
Kröll: Die Corona-Pandemie hat aber auch die Gesellschaft wesentlich verändert. Insbesondere durch die diversen Verordnungen des Gesetzgebers und die daraus resultierenden Einschränkungen im öffentlichen und privaten Bereich. Nun hast du im vergangenen Jahr zablreiche Menschen behandelt, die an COVID-19 erkrankt waren und hast einige davon auch sterben gesehen. Waren deiner Meinung nach, die diversen Anordnungen und Einschränkungen gerechtfertigt? Hätte man aus deiner Sicht etwas anders machen sollen?

Pongratz: Ich empfand die Einschränkungen als sehr sinnvoll und notwendig. Der Mensch ist ein soziales Wesen. COVID-19 aber isoliert, polarisiert und vereinsamt, da die Angst vor Ansteckung überall gegenwärtig ist. Besuch in sensiblen Bereichen wird nur in Ausnahmen (bei längerem Intensivaufenthalt, im Sterbeprozess) und nur unter strengen Hygienevorgaben erlaubt. Der Besuch von Angehörigen ist aber essenziell für das Wohlbefinden der PatientInnen. Eingeschränkte Wahrnehmung und Analgosedierung erschweren die Kommunikation oder machen sie unmöglich. Der Austausch mit dem sozialen Umfeld der Kranken fand bei uns hauptsächlich telefonisch statt. Die Angehörigen waren oft verzweifelt, aber sehr verständig. Sie sahen die Umstände ein, konnten sich aber in Wahrheit oft keine Vorstellung machen über das wahre Ausmaß der Erkrankung.

Wir als Pflegepersonen, die ständig um die Schwererkrankten herum sind, werden somit zu Bezugspersonen, zur Ersatzfamilie. Wir erleben Dankbarkeit, Sorgen, Ängste, Hoffnungen, aber auch beispielgebenden Mut, positive Kraft und Zuversicht.

In diesem Sinne würde ich mir einen angepassten, aber entsprechend möglichen Besuch wünschen zum Wohle der Familien und Kranken.

Kröll: Wenn du der österreichischen Bevölkerung etwas empfehlen dürftest, wie sie sich in der noch immer bestehenden Pandemie oder in einer späteren Epidemie oder Pandemie verhalten sollten? Welche Empfehlungen würdest du ibnen geben?

Pongratz: Die Erfahrung als Intensivschwester zeigt mir immer wieder deutlich, wie fragil Gesundheit sein kann und wie plötzlich und unvorbereitet Krankheit und Tod Menschen mitten aus dem Leben reißen. Krankheit kann unsere Handlungsfähigkeit verändern, diese stark einschränken. Dementsprechend gilt es die Gesundheit zu schützen durch Eigenverantwortung und Rücksichtnahme. Mein Apell ist, einen respektvollen Umgang miteinander zu wahren, mit Anstand, Geduld und Würde dem Mitmenschen gegenüber. Jeder wünscht sich das auch für sich. Jeder kann in Kleinem wie im Großen seinen persönlichen Beitrag dazu leisten. 
Lisa Pongratz, befragt von Wolfgang Kröll

Kröll: Liebe Lisa, vielen Dank für das Interview und dass du dir dafür Zeit genommen hast. 